\title{
Land Vehicle Navigation System Enhanced by Vibration Analysis
}

\author{
Tran Duc Tan ${ }^{\mathrm{a}}$, Luu Manh Ha ${ }^{\mathrm{a}}$, Nguyen Thang Long ${ }^{\mathrm{a}}$, Nguyen Phu Thuy ${ }^{\mathrm{a}}$, Huynh Huu Tue \\ ${ }^{a}$ Faculty of Electronics and Telecommunications, College of Technology, VNUH \\ ${ }^{\mathrm{b}} \mathrm{BacHa}$ International University \\ Hanoi, Vietnam \\ tantd@vnu.edu.vn, luumanhha85@gmail.com \\ longnt@vnu.edu.vn, thuynp@vnu.edu.vn, huynhuutue@bhiu.edu.vn
}

\begin{abstract}
Recent demand on the navigation systems is very high in many applications such as transportation and environment control. The inertial navigation system (INS) is not only suffering from errors caused by inertial sensors but also the vehicle dynamic. In this paper, the vibration analysis of the land-vehicle is applied for a special INS/GPS integration. The Strapdown INS (SINS) using two Kalman Filters (KF) has been built so that the system can be operated flexibly between feedforward and feedback modes in case of GPS outage. The experiment results show that this INS/GPS system can be used for practical applications.
\end{abstract}

Keywords: Vibration Analysis, Navigation, IMU, INS, Kalman

\section{INTRODUCTION}

Navigation and guidance are very important problems for marine, aeronautics and space technology. In such systems, Inertial Measurement Units (IMUs) are widely used as the core of the Inertial Navigation Systems (INS) [1]. The Inertial Navigation Systems (INS) has been widely used thanks to the strong growth of MicroElectronicMechanical System (MEMS) technology. The INS can provide us information about the position, velocity and attitude of the vehicle but it is suffering from errors caused by inertial sensors [2]. To reduce these errors, one of the most efficient methods is the combination of INS and GPS using Kalman filter. We can estimate the errors of both the INS and GPS in order to give more accurate information.

There is an extensive research on INS/GPS integration system to improve its performance [3]. The main contribution of this paper is to analyse the impact of vehicle's vibration and develop a scheme in which two Kalman filters operating in parallel have been applied flexibly in the navigation system. When the GPS signal is available, the INS/GPS runs in the feedback configuration. In case of GPS outage, the system will automatically switch to the feedforward configuration. After the GPS signal is reacquired, the system turns back to the feedback configuration. Switching between these two configurations can improve the performance of the system and reduce concurrently the disadvantages of both modes.

\section{VIBRATION ANALYSIS}

In this study, as for the INS system we have used the IMU BP3010 which consists of three low cost ADXRS300 gyros and three low cost heat compensated ADXL210E accelerometers [4]. The measurements are realized by IMU's micro-controllers and output data are transmitted out via RS232 interface. The unit transmits output data as angular incremental and velocity incremental values in serial frames of 16 bytes at the frequency of $64 \mathrm{~Hz}$.

There are two kinds of noise in the INS: deterministic and stochastic errors. The methods to eliminate these noises have been reported in [3]. However, the accuracy of the navigation system is also affected by vibration caused by vehicle's engine. In this paper, we have determined successfully the characteristics of this vibration noise by analyzing the Soft-Time Fourier Transform (STFT) of the experiment data. 
The vibration analysis can be divided into three phases: the vehicle stops when the engine is off, the vehicle stops when the engine is on, and the vehicle runs when the engine is on Figure 1 shows the velocity increment in vertical direction $(\mathrm{Az})$ within these phases. It is clear to see that the sensor is affected by instinct noise (Johson noise).

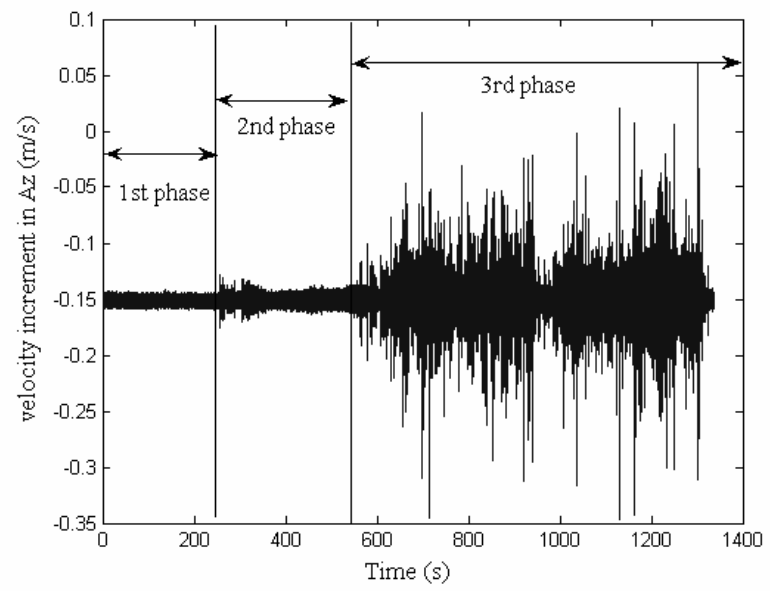

Fig. 1. Velocity increment in vertical direction $(\mathrm{Az})$

In our strapdown system, accelerometers and gyroscopes are fixed to body frame of the aircraft. Signals from these sensors are in the body-frame system which can be transformed to the navigation frame:

$$
\left[\begin{array}{c}
V_{N} \\
V_{E} \\
V_{D}
\end{array}\right]=C_{b}^{n}\left[\begin{array}{c}
\mathrm{V}_{\mathrm{x}}^{\mathrm{b}} \\
\mathrm{V}_{\mathrm{y}}^{\mathrm{b}} \\
\mathrm{V}_{\mathrm{z}}^{\mathrm{b}}
\end{array}\right]
$$

To obtain the STFFT, the function to be transformed is multiplied by a window function for a short period of time. Then, the Fourier transform is taken as the window is slid along the time axis. Thus, we have got a two-dimensional representation of the signal. Mathematically, this is written as:

$$
X(f, t)=\int_{-\infty}^{+\infty}\left[x(t) w(t-\tau)^{*}\right] e^{-2 j \pi \cdot f \cdot t} d t
$$

Where $w(t)$ is the window function, and $x(t)$ is the signal to be transformed. $\mathrm{X}(\mathrm{f}, \mathrm{t})$ is the Fourier transform of $\mathrm{x}(\mathrm{t}) \mathrm{w}(\mathrm{t}-\tau)$. This is a complex function representing the phase and magnitude of the signal over time and frequency.

The STFT can be shown by spectrogram in Fig. 2. The horizontal axis is in the range from 0 to $32 \mathrm{~Hz}$ which is suitable for navigation applications. The sampling frequency here is $64 \mathrm{~Hz}$ with note that the signal has gone through a low pass filter before. It is realized that the clear differences between the first phase and the second one of the experimental data are shown by arrows in Fig 3. This is vibration noise caused by vehicle's engine. This noise will be brought to the Inertial Navigation System (INS) and the accuracy of the output (positions, velocities, and attitudes) will be degraded. If these vibration signals are out of the navigation range, we can eliminate them by using digital filters. Otherwise, we can only reduce the impacts of these vibrations by mechanical techniques while assembling the Inertial Measurement Unit (IMU).

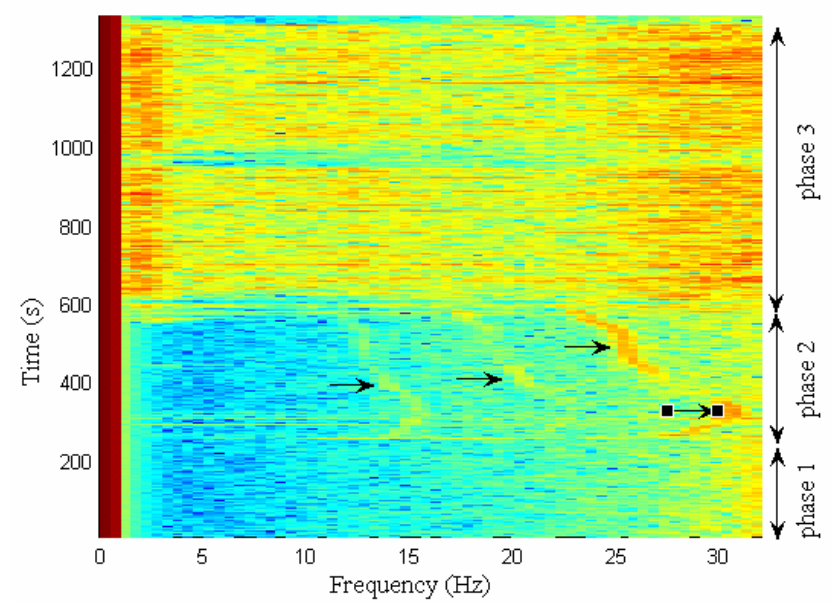

Fig. 2. Spectrogram of the $A Z$ sensor

Figure. 3 is the Power Spectrum Density (PSD) of $\mathrm{Az}$ acceleration sensor. In the frequency range of 0.3 and $32 \mathrm{~Hz}$, the spectrum density is nearly constant and can be assumed to be background noise. However, when we focus to the range of low frequency from 0 to $0.3 \mathrm{~Hz}$, we can realize the present of flicker noise that cause drifts at the system outputs. These kinds of noise can be treated effectively by using optimal Kalman Filters (KF). 


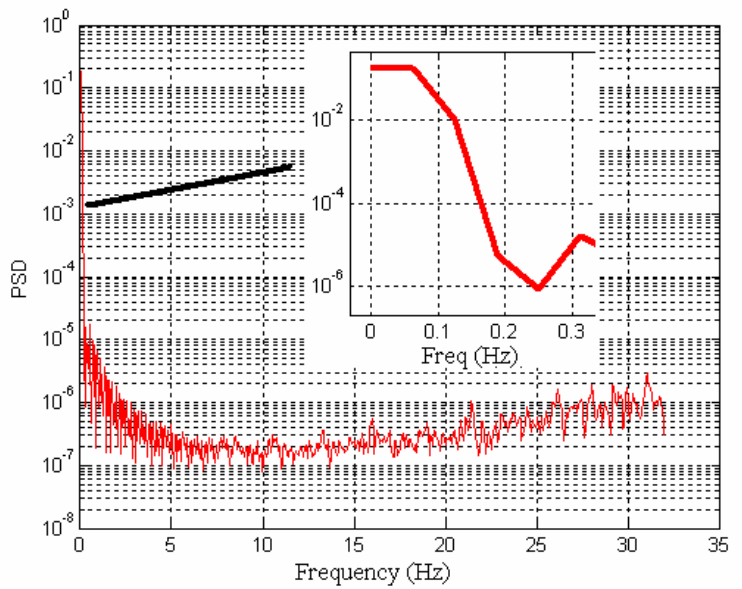

Fig. 3. Power Spectrum Density of AZ acceleration sensor

Figure 4 shows the PSD of three phases, concurrently. The differences between the first phase and the second one are quite small except the signal in the range of 20 and $35 \mathrm{~Hz}$. In the third phases, the navigation signal is in the range of 5 and $15 \mathrm{~Hz}$. Thus, we can utilize several kinds of low pass filters to reduce the vibration noises.

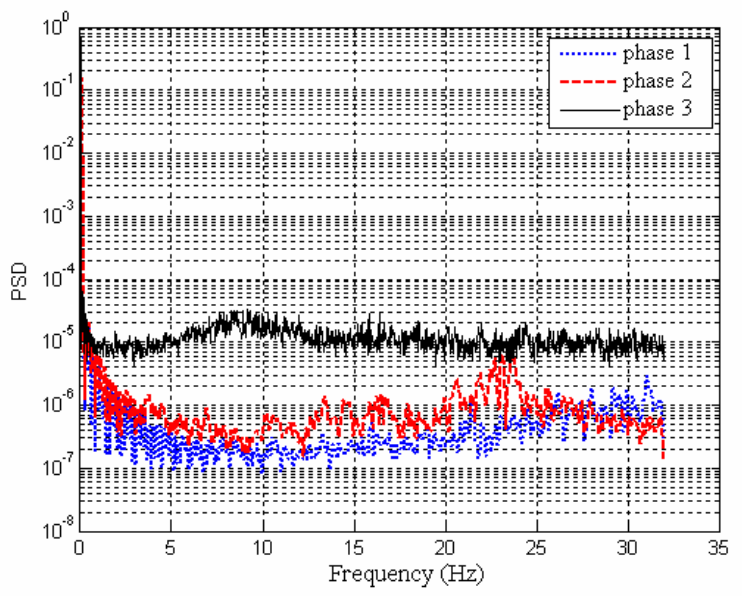

Fig. 4. Power Spectrum Density of AZ acceleration sensor

Figures. 5.a and 5.b are spectrograms of the AX and AY accelerometer in which we can applied similar processes to characterize these acceleration components.

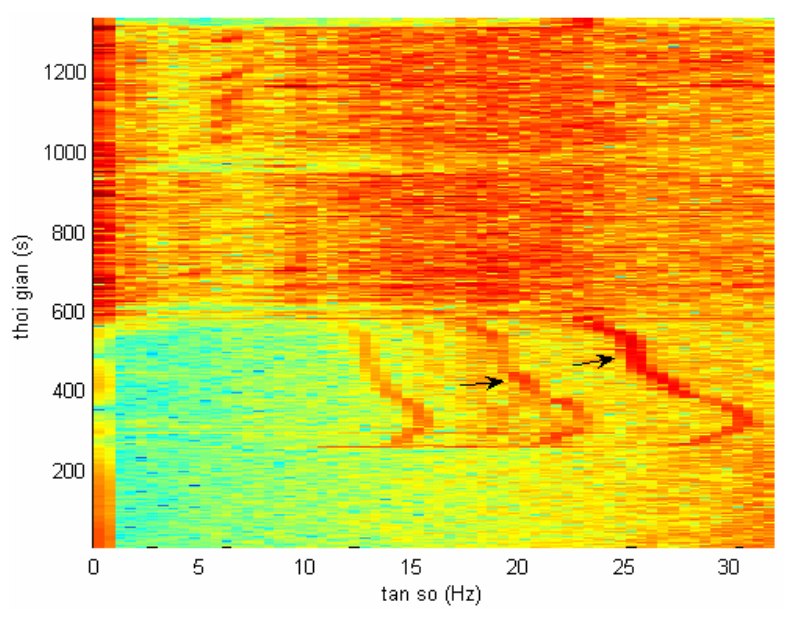

(a)

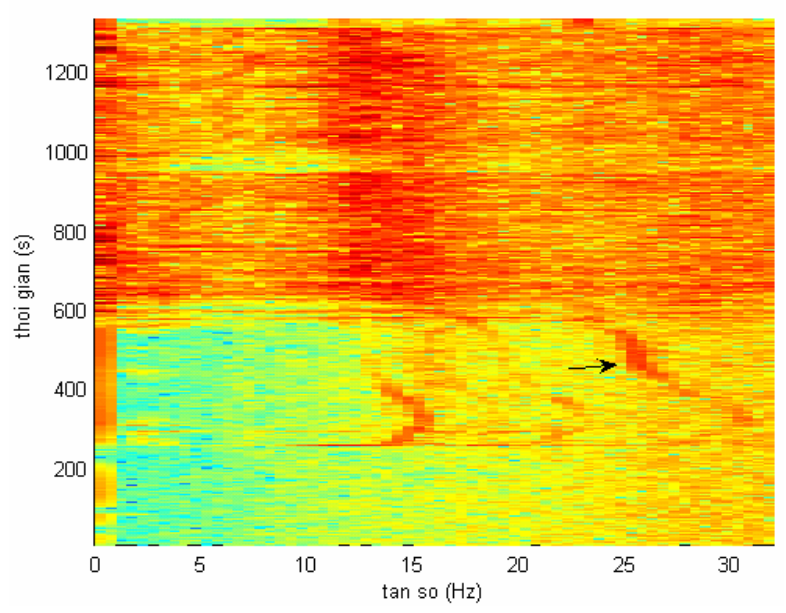

(b)

Fig. 5. Spectrogram of the $A X(a)$ and $A Y(b)$ sensors

\section{APPLICATION TO INS/GPS SYSTEM}

After characterizing the noises, the information of these noises is applied to the KF based MEMSINS/GPS integration module. Figure 6 illustrates an open loop (or feedforward) configuration. Its advantage is that provides a rapid filter response. Alternatively, the configuration in Figure 7 is a closed loop one. This configuration is more complex than the open loop one but it can provide better performance in the exist of nonlinear effects.

The aim of this section is to develop of a specific scheme for INS/GPS integration that can be used in the case that GPS signal gets lost frequently. The 
integration system based on two parallel Kalman filters is developed and tested. The first Kalman block is applied to obtain a fast convergence due to its small state. Thus, the velocities and position of the vehicle can be quickly corrected. The second KF has the ability to accommodate and estimate the attitude errors. Furthermore, the INS/GPS system can switch between feedforward and feedback schemes depending on GPS environments. The INS/GPS error estimation scheme is shown in Figure 7. The INS error equations are used as a system model and the measured input data fed to the filter are the differences between the INS and GPS [5]. When GPS data are not available, the Kalman filter works in prediction mode and the INS/GPS system switch to feedforward scheme.

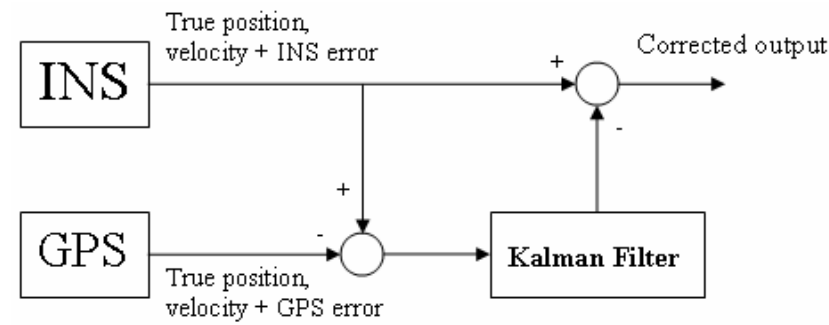

Fig. 6. Feedforward configuration

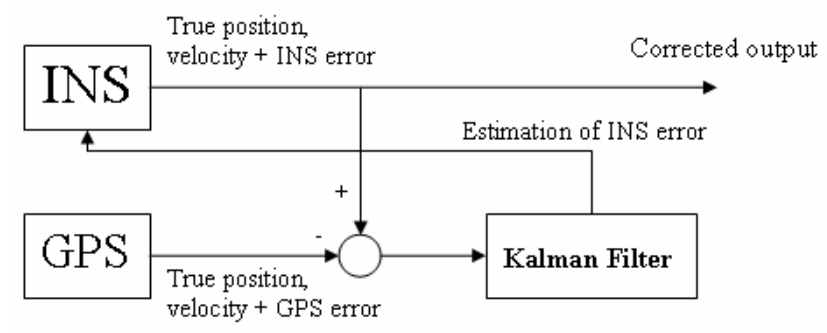

Fig. 7. Feedback configuration

In discrete form, any linear system can be described as:

$$
x_{k}=A_{k, k-1} x_{k-1}+G_{k, k-1} w_{k-1}
$$

Where $A_{k, k-1}$ is a $(n \times n)$ transition matrix, $G_{k, k-1}$ is an $(n \times r)$ input matrix, and $\mathrm{w}_{\mathrm{k}-1}$ is $(r \times 1)$ input noise. We can derive these matrix based on the INS error equations

And the measurement model:

$$
z_{k}=H_{k} x_{k}+v_{k}
$$

Where $\mathrm{z}_{\mathrm{k}}$ is a $(m \times 1)$ measurement vector, $\mathrm{H}_{\mathrm{k}}$ is a $(m$ $x n)$ design matrix, and $\mathrm{v}_{\mathrm{k}}$ is $\left(\begin{array}{l}m \times 1 \\ x\end{array}\right)$ measurement noise.

In the first block (KF1), a conventional Kalman filter with a reduced system model is utilized for the INS velocity error estimation. The measurement vector here is velocity differences between GPS and INS. Estimated INS velocity errors are compensated in the system output.

In the KF2, the estimation of INS errors is performed in order to improve estimation accuracy. The measurement vector here are velocity and position differences between GPS and INS. There are eight such states $\left(x_{k}\right)$ which consist of attitude errors $\left(T_{n}, T_{e}\right)$, velocity errors ( $\left.e_{\mathrm{VN}}, e_{\mathrm{VE}}, \mathrm{e}_{\mathrm{VD}}\right)$, and drift terms $\left(\mathrm{G}_{\mathrm{bx}}, \mathrm{G}_{\mathrm{by}}\right.$, $\mathrm{G}_{\mathrm{bz}}$ ). The INS errors are used to correct the elements of the transformation matrix $C_{b}^{N}$ and the quaternion. Estimated gyro drifts are also taken into account in the SINS navigation scheme. The transition matrix is:

$$
A_{k, k-1}=I+\left[\begin{array}{cccccccc}
0 & 0 & 0 & 0 & 0 & h_{N} C_{11} & h_{N} C_{12} & h_{N} C_{13} \\
0 & 0 & 0 & 0 & 0 & h_{N} C_{21} & h_{N} C_{22} & h_{N} C_{23} \\
0 & -D v d & 0 & 0 & 0 & 0 & 0 & 0 \\
D v d & 0 & 0 & 0 & 0 & 0 & 0 & 0 \\
0 & 0 & 0 & 0 & 0 & 0 & 0 & 0 \\
0 & 0 & 0 & 0 & 0 & -h_{N} \beta & 0 & 0 \\
0 & 0 & 0 & 0 & 0 & 0 & -h_{N} \beta & 0 \\
0 & 0 & 0 & 0 & 0 & 0 & 0 & -h_{N} \beta
\end{array}\right]
$$

Where $I$ is unit matrix $(8 \times 8), D v d$ is the velocity increment in Down direction of the navigation frame (north, east, down), $\beta$ is one of parameters of the correlation function, and $\mathrm{h}_{\mathrm{N}}$ here is $0.015625 \mathrm{~s}$ (equivalent to $64 \mathrm{~Hz}$ ).

In the case of the GPS signal blockage, positioning is provided by the INS until GPS signals are reacquired. During such periods, navigation errors increase rapidly with time due to the time-dependent INS error behavior. In these cases, we sometime utilized land vehicle motion behavior to prevent INS from the error accumulation. The equation derived from behavior of a 
land vehicle will compensate the GPS's measurements. In this paper, the velocity and height constraints of the land vehicle have been utilized. They can provide the virtual measurements to aid the IMU.

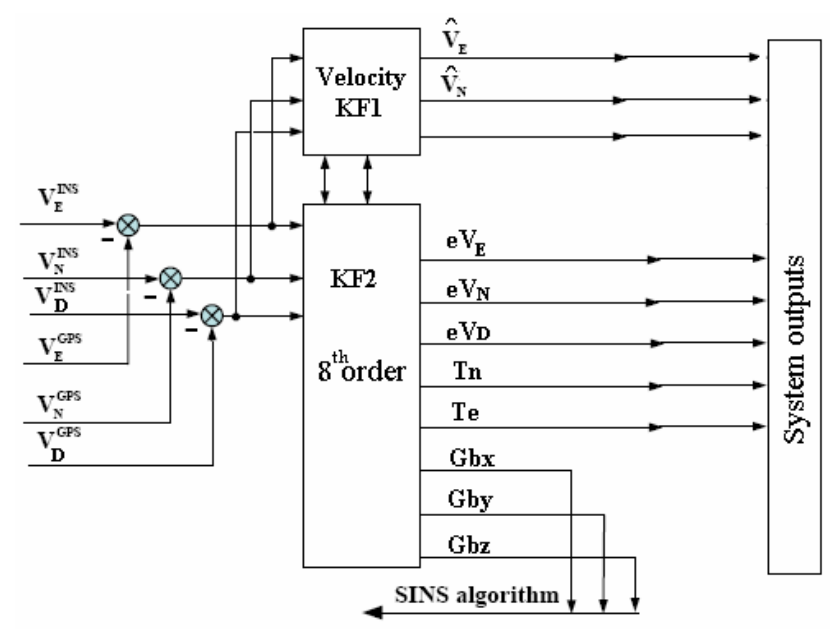

Fig. 8. The integration configuration

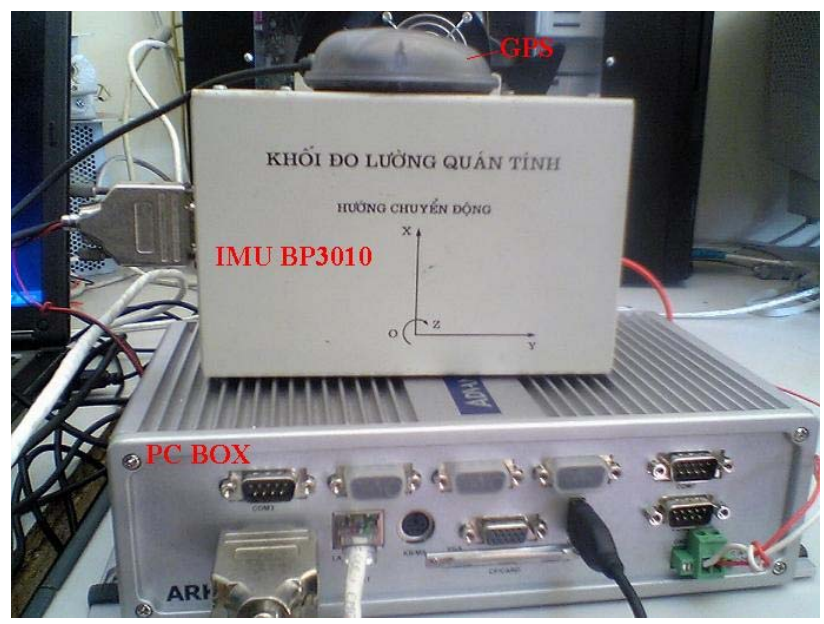

Fig. 8. Hardware for the proposed navigation system

We can only correct the attitude of the IMU using the attitude errors predicted by the state matrix. This corrected attitude forms a part of the whole system. To take the in-motion alignment of the navigation system, we use the heading from GPS or the external heading measurements such as magnetometer for the attitude computations.

Figure 8 is the hardware for the proposed navigation system.

\section{EXPERIMENTAL RESULTS}

In order to prove the efficiency of the new scheme, a experiment trajectory is performed in which GPS signal was lost within 100 seconds. For this experiment, the system was installed in a mini vehicle [6,7]. The IMU is placed inside of the vehicle and the GPS is placed outside of the vehicle. The INS computations and its integration with the GPS are carried out on a commercial PC box. Initially the vehicle was at rest, when the engine is on, for about 30 seconds. This stationary data was used for calibration and alignment purposes. The update from the INS was taken every $0.015625 \mathrm{~s}$, the GPS update was taken every $1 \mathrm{~s}$ and the KF was run every $0.5 \mathrm{~s}$ to achieve better accuracy. We can see that the IMU provides navigation information with high frequency in between GPS updating. At each $0.015625 \mathrm{~s}$, the vehicle velocity, position, attitude and quaternion are updated.

In the case that the GPS receiver loses its signal for 100 seconds, the INS can continue to compute the position. Figure 9.a presents the trajectory using feedback configuration of KF compared with the values measured with the GPS unit. It can be seen that outputs of KF, the solid curve, can not follows well. In contrast, the combination of the two configurations can give much better results as shown in Figure 9.b. In this combined structure, the feedback KF is utilized when the GPS signal is available and the feedforward KF is applied when GPS is outage.

To determine more precisely the quality of the navigation system, the system is examined in another trajectory in which the vehicle was driven for 21 minutes. Initially the vehicle was at rest and the engine is on, for about 600 seconds. Figure 10 illustrates the comparison of the rolls between the systems with and without vibration suppression. In the first 600 seconds, it is clear to see that the system with vibration suppression could provide the better results. In the latter period, the roll given by the proposed system is also seemed to be more precise. 


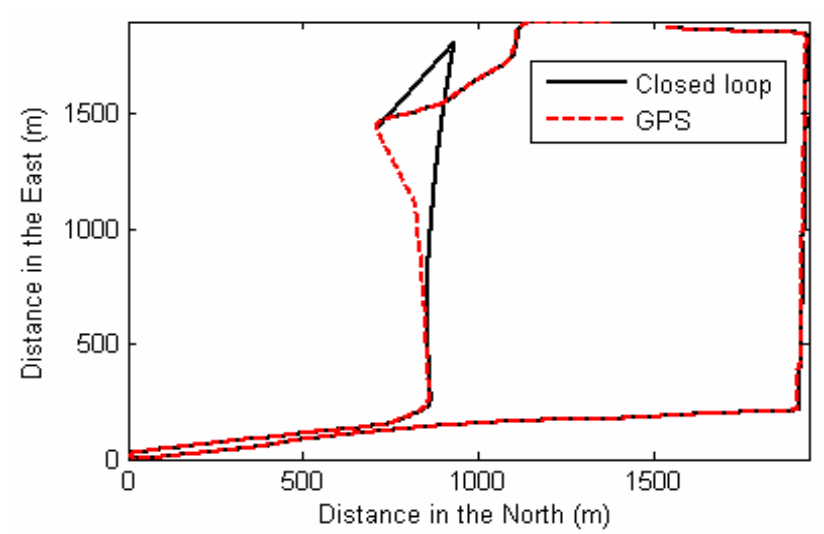

(a)

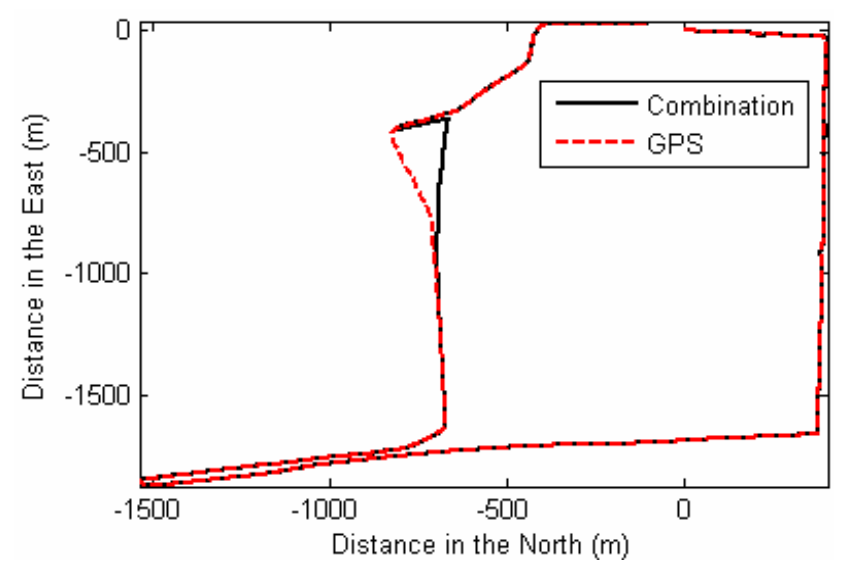

(b)

Fig. 9. Comparison of the feedback configuration (a) and the combined one (b) in the case of GPS outage

The graphs for velocity computed and corrected by the Kalman filter are given in Fig. 11. We can see that the un-aided INS deviates from the ideal velocity by a large quantity. If the integration system is supported by $\mathrm{KF}$, the output $\mathrm{Vn}$ is around $0 \mathrm{~m} / \mathrm{s}$. It means that our $\mathrm{KF}$ could give the exact correction.

\section{CONCLUSION}

This paper has succeeded in specifying the vibration noises caused by land-vehicle engine, which is a necessary step when applying error-processing algorithms for the INS. The extracted results will be used as the parameters in Kalman filters for the INSGPS integrated system. In this paper, the new scheme using two parallel Kalman filters was proposed to be used in order to enhance the quality of a combined GPS and INS system. The accuracy of navigation is also improved by flexible switching between feedback and feedforward configurations in the case of GPS outage. Our future work will concentrate in the in-flight calibration and alignment algorithms that extend the present error models of the INS system.
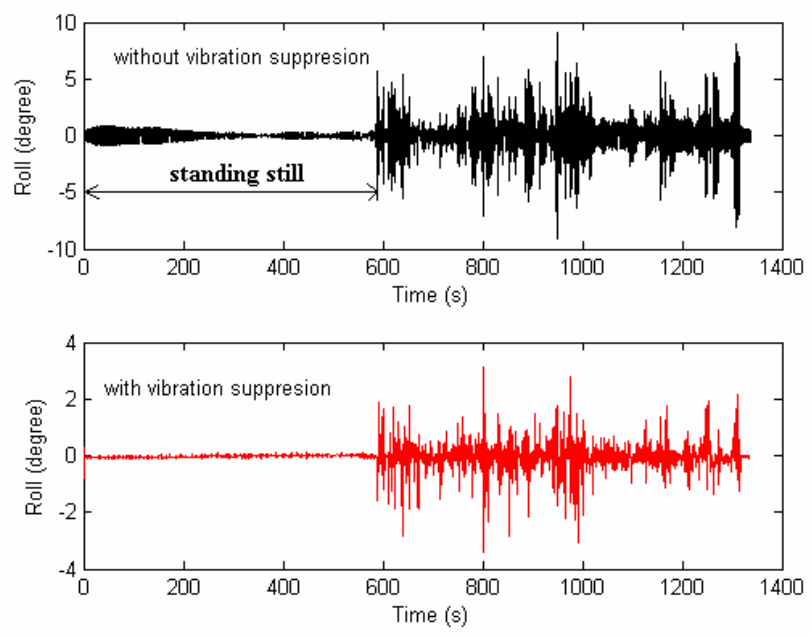

Fig. 10. Roll angles of the systems with and without vibration suppression

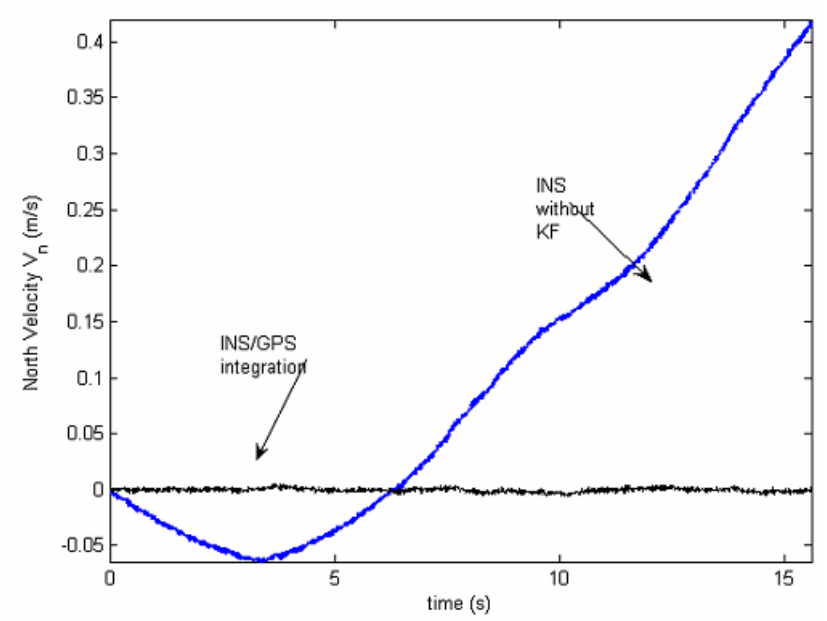

Fig. 11. The north velocity of the stand still IMU in two cases: with and without $\mathrm{KF}$

\section{ACKNOWLEDGMENT}

This work is supported by the QC- 08.13 project of Coltech, VNUH. 


\section{REFERENCES}

[1] Vikas Kumar N, Integration of Inertial Navigation System and Global Positioning System Using Kalman Filtering, M.Tech. Dissertation, Indian Institute of Technology, Bombay, July 2004.

[2] Oleg S. Salychev, Applied Inertial Navigation: Problems and Solutions, BMSTU Press, Moscow Russia, 2004.

[3] Tran Duc Tan, Luu Manh Ha, Nguyen Thang Long, Nguyen Phu Thuy, Huynh Huu Tue, Performance Improvement of MEMS-Based Sensor Applying in Inertial Navigation System, Research - Development and Application on Electronics, Posts, Telematics \& Information Technology Journal, No.2, pp. 19-24, 2007.

[4] Georey J.Bulmer, In MICRO-ISU BP3010 An OEM Miniature Hybrid 6 Degrees-Of-Freedom Inertial Sensor Unit. Gyro Symposium, Stuttgart 16th-17th September, 2003.

[5] Wang, J., Lee, H.K., Rizos, C., GPS/INS Integration: A Performance Sensitivity Analysis, University of New South Wales, Sydney.

[6] Gyro, Accelerometer Panel of the IEEE Aerospace, and Electronic Systems Society. Draft recommended practice for inertial sensor test equipment, instrumentation, data acquisition and analysis. In IEEE Std Working Draft P1554/D14.

[7] Panzieri, S., Pascucci, F., Ulivi, G., "An Outdoor navigation system using GPS and Inertial Platform”, IEEE ASME Transactions on Mechatronics, Vol. 7.(2002).

\section{AUTHORS' BIOGRAPHY}

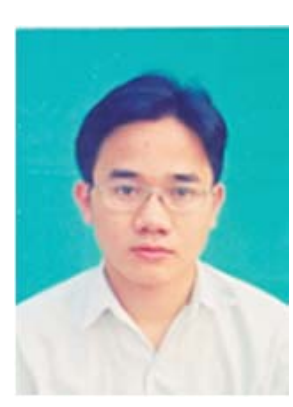

Tran Duc Tan was born in 1980. He received his B.Sc and M.Sc degrees respectively in 2002 and in 2005, both at the College of Technology (COLTECH), Vietnam National University - Hanoi, Vietnam (VNU), where he has been a lecturer since 2006. He is currently completing his $\mathrm{PhD}$ thesis at COLTECH, VNUH. He is author and coauthor of several papers on capacitive accelerometers, silicon micromachined gyroscopes, and piezoresistive accelerometers. His present

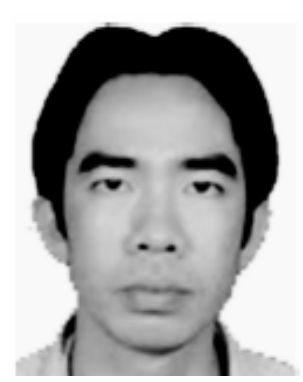
research interest is in the development of MEMS-based inertial navigation systems.

Nguyen Thang Long received the M.S. degree from the International Institute of Materials Science, Hanoi University of Technologies, Hanoi, Vietnam in 1998, and the Doctor of Engineering degree from the
University of Twente, Enschede, The Netherlands, in 2004. He has worked as a Lecturer with the Faculty of Electronics and Telecommunications, College of Technology, Hanoi National University, since 2004. His main activities are related to design and application of MEMS sensors. He has been involved in several projects such as designing of the patient monitoring system and integrations of inertial MEMS sensors and GPS for navigation.

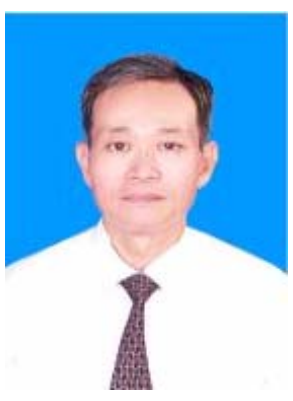

Nguyen Phu Thuy received his PhD degree in 1979 at Charles University, Prague, Czechoslovakia. Since 1980, he has been a faculty member of the Vietnam National University, Hanoi (VNUH). He has also been associated to International Training Institute for Materials Science (ITIMS) since 1992 as senior researcher. In 2005, he was nominated Dean of the Faculty of Electronics and Telecommunications, College of Technology, VNUH. He is author and coauthor of more than one hundred papers published in professional journals and international conferences. His research interests cover magnetic materials and MEMS-based sensors with applications.

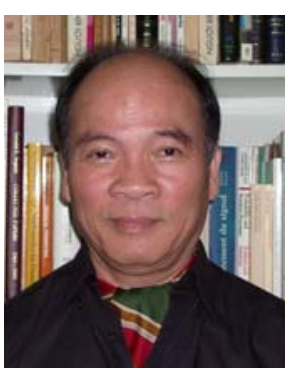

Huu Tue Huynh received his Sc.D. from Laval University in 1972, where he had been a Professor of the Department Electrical and Computer Engineering since 1969. He left Laval in 2004 to become the Chairman of the Department of Information Processing of the College of Technology, Vietnam National University, Hanoi and recently nominated Rector of Bac Ha International University. He has been an invited professor at l'INSA (Lyon, France) in 1972, ENST (Paris, France) in 1980, l'Universite de Rennes (France) in 1982, Concordia University (Montreal, Canada) in 1985, Ecole Polytechnique (Montreal, Canada) in 1986, l'Universite de Sherbrooke (Sherbrooke, Canada), in 1990, CEPHAG (Grenoble, France) in 1995. In 1984, he was an invited guest of Bell Lab (Neptune, N.J. USA). He is author and coauthor of more than one hundred papers published in professional journals and international conferences; he is also coauthor of two books, "Systemes non-lineaires" (Gordon \& Breach 1972) and "Simulations stochastiques et applications en Finances avec des Programmes Matlab" (Economica, 2006); the English version of the second book will be published by Wiley in 2008. His research interests cover stochastic simulation techniques, information processing, fast algorithms and architectures with applications to digital communications 